See Article page XXX.

\section{Commentary: Should I stay (at primary valve center) or should I go (transfer to comprehensive valve center)?}

\author{
Frank A. Baciewicz, Jr, MD
}

The Clash asked in their classic 1982 rock anthem, "Should I Stay or Should I Go." The article "Surgical Treatment of Infective Endocarditis at Comprehensive Versus Primary Valve Centers" by Squiers and colleagues ${ }^{2}$ similarly asked if patients with primary infective endocarditis "should stay" at primary valve centers (PVCs) or "go" (transfer to) comprehensive valve centers (CVCs).

CVCs were defined as hospitals with surgeons capable of performing valve-sparing aortic root procedures (none performed for endocarditis in this series), procedures for aneurysmal disease, mitral valve repair, multiple valve procedures, and reoperative surgery, with expertise in echocardiography and 24/7 intensivist coverage for the cardiac surgery intensive care unit. ${ }^{3}$

Within the Baylor, Scott, and White Health Care Systems, the 2 CVCs had a $6.2 \%$ 30-day mortality, whereas the 6 PVCs had a $13.0 \%$ operative mortality for $2: 1$ propensity-matched initial endocarditis operations. Approximately $70 \%$ of the patients in each group had active endocarditis, and the CVC cohort received more Bentall procedures (60.4 vs 21.7) and mitral valve repairs (50.4 vs 26.3).

The more favorable CVC results were ascribed to the institutions' multidisciplinary decisions whether to offer surgical intervention and to opt for early surgical intervention with radical debridement. These factors may have contributed, but without knowing the total number of

\footnotetext{
From the Division of Cardiothoracic Surgery, Michael and Marian Ilitch Department of Surgery, Wayne State University School of Medicine, Detroit, Mich. Disclosures: The author reported no conflicts of interest.

The Journal policy requires editors and reviewers to disclose conflicts of interest and to decline handling or reviewing manuscripts for which they may have a conflict of interest. The editors and reviewers of this article have no conflicts of interest.

Received for publication Sept 24, 2021; revisions received Sept 24, 2021; accepted for publication Sept 28, 2021.

Address for reprints: Frank A. Baciewicz, Jr, MD, Division of Cardiothoracic Surgery, Michael and Marian Ilitch Department of Surgery, Wayne State University School of Medicine, Harper Hospital, 3990 John R, Detroit, MI 48201 (E-mail: fbaciewi@dmc.org).

J Thorac Cardiovasc Surg 2021; $\mathbf{\square}: 1-2$ $0022-5223 / \$ 36.00$

Copyright (c) 2021 by The American Association for Thoracic Surgery https://doi.org/10.1016/j.jtcvs.2021.09.046
}

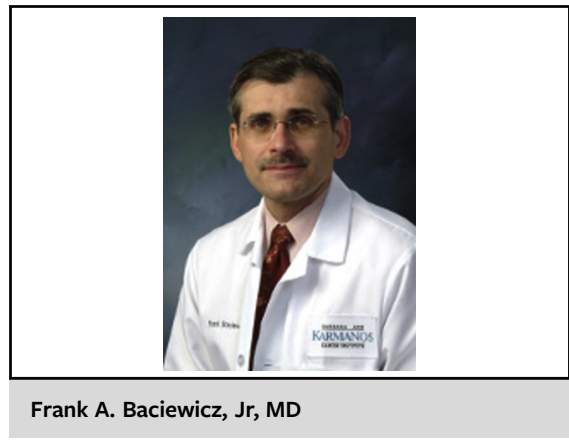

CENTRAL MESSAGE

A healthcare system demonstrated lower mortality for primary endocarditis surgery at their high-volume cardiac surgery centers compared with the systems' low-volume cardiac surgical centers.

patients evaluated and turned down for surgical intervention, the value of a second opinion or multidisciplinary approach in selecting a successful operative cohort is unknown. There are multiple mortality score calculations for endocarditis valve surgery, but they do not universally tabulate points for abscess, tricuspid valve, or multiple valve involvement. It might be informative to know how particular calculations stacked up against the CVC multidisciplinary decision or the clinician's decision at the PVC. Did a calculated Society of Thoracic Surgeons or Association for the Prevention of Infective Endocarditis score influence the CVC or PVC decision makers? Could a calculated mortality score be as good as a clinical decision?

Likewise, predicting the extent of debridement preoperatively cannot be done by a committee. The surgeon often finds an unexpected abscess or severely damaged tissues at operation. The 30-day mortality for propensity-matched active and treated endocarditis outcomes versus only active endocarditis outcomes (CVC 6.2\% mortality for the combination and $6.4 \%$ for active only, PVC $13.0 \%$ mortality for the combination and $14.8 \%$ for active only) was identical for both PVC and CVC centers. It is hard to appreciate any change in outcome with earlier surgical intervention in the "active" CVC endocarditis cohort. 
The 2 CVC hospitals averaged 32 endocarditis cases per year over the 2014-2020 study, whereas the PVC hospitals averaged less than 3.5 cases per year. The disparity in operative volumes probably explains the difference in outcomes, and it would be interesting to check outcomes for nonendocarditis valve operations or coronary artery bypass grafting procedures at the $\mathrm{CVC}$ versus the PVC hospitals. I would predict that the differences in outcomes might exist for other cardiac procedures. Additionally, was there a difference in outcomes between the 2 CVC hospitals? CVC hospital 1 performed more than 6000 valve cases over this study period, whereas CVC hospital 2 performed 1300 cases. Did any of the PVCs have performance similar to CVC hospital 1 or CVC hospital 2?

Do the CVC and PVC have data on 30-day and long-term outcomes for patients treated medically (without surgical intervention)? Multidisciplinary care at CVCs might be expected to demonstrate improved survival in the medical and surgical cohorts.

Was any of the operative mortality due to a failure to rescue secondary to absence of the $24 / 7$ intensive care unit staffing at the PVCs? Certainly, this is a factor that could be changed at the PVC.

My impression is that the CVC versus PVC difference in outcomes is related to a combination of superior support services, top-notch surgical and medical expertise, and advanced imaging available at high-volume centers. I expect further review by other healthcare systems will confirm improved primary endocarditis outcomes at higher-volume cardiac centers. Confirming this article's results and investigating its queries will provide an answer to the question "Should surgical endocarditis patients stay (at PVC) or should they go (be transferred to CVC)"?

\section{References}

1. The Clash. Should I Stay or Should I Go. Epic Label; 1982.

2. Squiers J, et al. Surgical treatment of infective endocarditis at comprehensive venous primary valve centers. J Thorac Cardiovasc Surg. 2021. XX:XX.

3. Nishimura RA, O'Gara PG, Bavaria JE, Brindis RG, Carroll JD, Kavinsky CJ, et al. 2019 ASTS/ACC/ASE/SCAI/STS expert consensus systems of care document: a proposal to optimize care for patients with valvular heart disease: a joint report of the American Association for Thoracic Surgery, American College of Cardiology, American Society of Echocardiography, Society for Cardiovascular Angiography and Interventions, and Society of Thoracic surgeons. J Thorac Cardiovasc Surg. 2019;157:e327-54. 